\title{
Screening of Auricularia auricula strains for strong production ability of melanin pigments
}

\author{
Yu ZOU ${ }^{1 *}, K^{\prime} M^{1}$
}

\begin{abstract}
Melanin pigments have great application value and development potential in food industry to use as nature functional food colorants. In initial study, twenty-two Auricularia auricula strains were screened for stronger production ability of melanin pigments by solid culture. Three A. auricula strains (RF201, QD2 and QD6) with higher pigment production capacity were selected for further study through submerged culture supplementing $1 \mathrm{~g} / \mathrm{L}$ l-tyrosine. The maximal pigment yields of A. auricula RF201, QD2 and QD6 were 493.9, 367.6 and $318.5 \mathrm{mg} / \mathrm{L}$, respectively. Among three strains, A. auricula RF201 possessed the strongest production ability of melanin pigments. The present study indicated that $A$. auricula RF201 could be used as potential excellent producer of melanin pigments.
\end{abstract}

Keywords: screening; Auricularia auricula; production ability; melanin pigments.

Practical Application: A. auricula RF201 can be used as potential excellent producer of nature melanin pigments.

\section{Introduction}

Melanin pigments are the natural black-brown polymers with high relative molecular weights (Chen et al., 2008). They are formed from the oxypolymerization of phenols through tyrosine oxidases in plant, animal, bacterium and fungus (Dalfard et al., 2006). Melanin pigments obtained from various sources have same physical and chemical characteristics, such as solubility and optical absorption property. Moreover, melanin pigments possess many biological activities, including oxidation resistance (Dong \& Yao, 2012; Liu et al., 2011; Tu et al., 2009), anti-aids effect (Zou et al., 2015), and immunoregulatory function (Wu et al., 2008). Therefore, melanin pigments have great application value and development potential in food industry to use as nature functional food colorants.

A. auricula is widely distributed in East and Southeast Asia. Its fruiting body called black fungus in China is used as an edible mushroom for centuries (Sun et al., 2016). A. auricula dark-colored fruiting body contains abundant melanin pigment which is popular in China as one of black foods. Melanin pigments are regarded as major bioactive substance in black foods (Tu et al., 2009; Wang et al., 2006). However, extracting processes of melanin pigments from tissues and cells of $A$. auricula fruiting body are costly and cumbersome. Furthermore, the growing cycle of A. auricula fruit body on solid culture medium is long (Wu et al., 2006).

A. auricula may also produce melanin pigments by submerged fermentation culture (Zou \& Hou, 2017). However, production abilities of melanin pigments are lower for most of A. auricula strains. Therefore, it is very important to screen high melanin-producing fungal strain. This research was conducted to screen high melanin-producing fungal strain from twenty-two
A. auricula strains for the potential use as an effective producer of natural melanin pigments.

\section{Materials and methods}

\subsection{Strains}

The fungal strains A. auricula NKY01, F1, F2, F3, F4, F5, QD1, QD2, QD4, QD6, QD7, QY8582, QY8583, QY8584, RF173, RF201, RF204, RF218, RF230, RF231, RF233 and RF235 were obtained from the Institute of Shanxi Edible Mushroom (Ankang, China). Mycelia kept on PDA slant were inoculated and incubated at $28^{\circ} \mathrm{C}$ for 7 days.

\subsection{Experimental design}

In initial experiment, $A$. auricula strains were screened by solid culture. PDA plate was inoculated with mycelium and incubated at $28^{\circ} \mathrm{C}$ for 7 days. Then, $5 \mathrm{~mm}$ diameter mycelium disc obtained from PDA plate was transferred to new PDA plate and incubated at $28{ }^{\circ} \mathrm{C}$ for 7 days. After that, visual colors of colony center and growth rate of mycelium were measured to screen stronger melanin-producing fungal strains.

A. auricula strains possessed stronger production ability of melanin pigments were further screened by submerged culture. Fungus was transferred to seed medium (potato dextrose broth medium) with $5 \mathrm{~mm}$ diameter disc from PDA plate to produce inoculums. Five discs were inoculated to $50 \mathrm{~mL}$ seed medium in $250 \mathrm{~mL}$ triangular flask and then incubated at $28^{\circ} \mathrm{C}$ on a shaking table at $100 \mathrm{rpm}$ for 5 days. Inoculums $(8 \%, \mathrm{v} / \mathrm{v})$ were transferred into $250 \mathrm{~mL}$ triangular flask contained $50 \mathrm{~mL}$ fermentation medium (potato dextrose broth medium supplementing $1 \mathrm{~g} / \mathrm{L}$ l-tyrosine as substrate). Afterward, it was cultivated at $28^{\circ} \mathrm{C}$ for 8 days at rotation time of $100 \mathrm{rpm}$. Finally, pigment yield and 
dry weight of mycelium were determined to obtain the strongest melanin-producing fungal strain.

\subsection{Visual colors of colony center}

Visual colors of colony center were monitored by a Minolta CR-400 colorimeter (Osaka, Japan). Visual colors in Hunter Lab color system were indicated as $L^{*}, a^{*}$ and $b^{*}$ values which respectively represented from darkness to whiteness, from greenness to redness and from blueness to yellowness.

\subsection{Growth rate of mycelium}

Growth rate of mycelium was determined according to method of Xie et al. (2010). The different colony diameters in vertical direction were monitored with the ruler. The Equation 1 was used to calculate growth rate of mycelium.

$$
Y=\left(X_{1}-X_{2}\right) / X_{3}
$$

Where $Y$ was growth rate, $X_{1}$ was final colony diameter, $X_{2}$ was initial colony diameter $(5 \mathrm{~mm})$, and $X_{3}$ was incubation time.

\subsection{Pigment yield of A. auricula}

Measurement of pigment yield was performed as described by Zou et al. (2010). Fungal fermentation broths were filtered to eliminate mycelia and impurities. The liquid was adjusted using $2 \mathrm{M} \mathrm{HCl}$ to $\mathrm{pH} 1.5$ to precipitate and separate pigment by centrifugation for $15 \mathrm{~min}$ at $8000 \mathrm{rpm}$. Precipitated pigment was respectively cleaned using ethyl acetate and ethanol. Then, melanin pigments were dissolved in $0.02 \mathrm{M} \mathrm{NaOH}$. Optical density of solution contained pigment at $400 \mathrm{~nm}$ was determined by Unico-2800 spectrophotometer (Princeton, USA).

\subsection{Dry weight of mycelium}

Mycelium biomass of $A$. auricula was measured through weighting dry mycelium amount. Fungal fermentation broths were filtered to obtain mycelia, and then mycelia were cleaned with deionized water and dried to achieve constant weight at $110^{\circ} \mathrm{C}$.

\subsection{Statistical analysis}

Experimental result was showed as mean \pm standard deviation and significant difference $(p<0.05)$ among different strains were analyzed by Fisher's F-test.

\section{Results and discussion}

\subsection{Mycelium growth and pigment secretion of A. auricula in PDA plate}

Mycelium growth and pigment secretion of twenty-two A. auricula strains in PDA plates are shown in Figure 1. All A. auricula mycelia used in this experiment grew well in PDA plates. As indicated in Table 1, average growth rate of twenty-two A. auricula strains was $5.6 \mathrm{~mm} /$ day. A. auricula RF230 exhibited the fastest growth rate of $9.3 \mathrm{~mm}$ /day. On the contrary, A. auricula $\mathrm{F} 3$ showed the slowest growth rate $(3.9 \mathrm{~mm} /$ day $)$. The experimental result showed that there was significant difference $(p<0.05)$ in growth rates among twenty-two $A$. auricula strains. It appeared that different strains from the same fungus obviously had different growth rate of mycelia in PDA culture media, which agreed with results reported by Xie et al. (2010).

Determination results (Table 1) from the colorimeter exhibited that the there was significant difference $(p<0.05)$ in $L^{*}, a^{*}$ and $b^{*}$ values of twenty-two $A$. auricula strains in Hunter Lab color system. Visual colors of colony center varied greatly
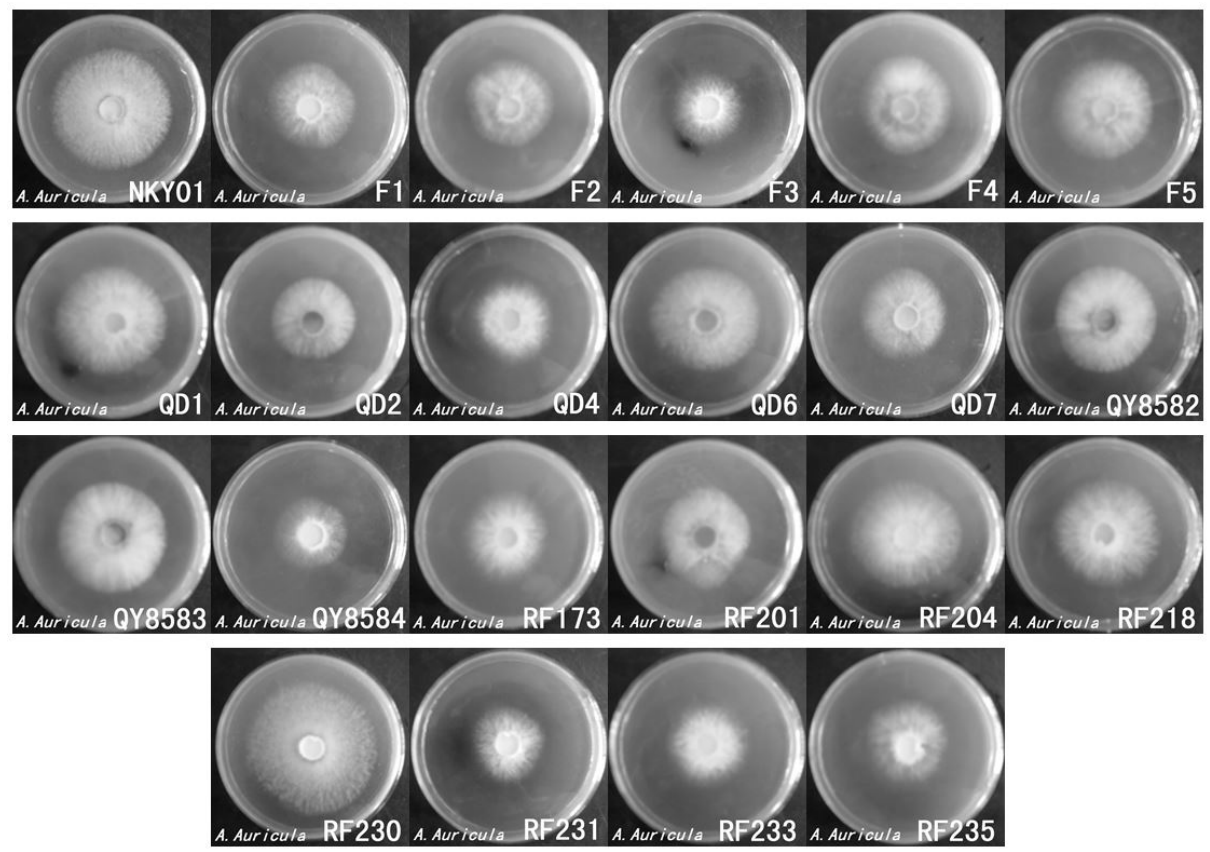

Figure 1. Mycelium growth and pigment secretion of A. auricula strains in PDA plates. 
Table 1. Growth rate of mycelium and visual colors of colony center of A. auricula strains.

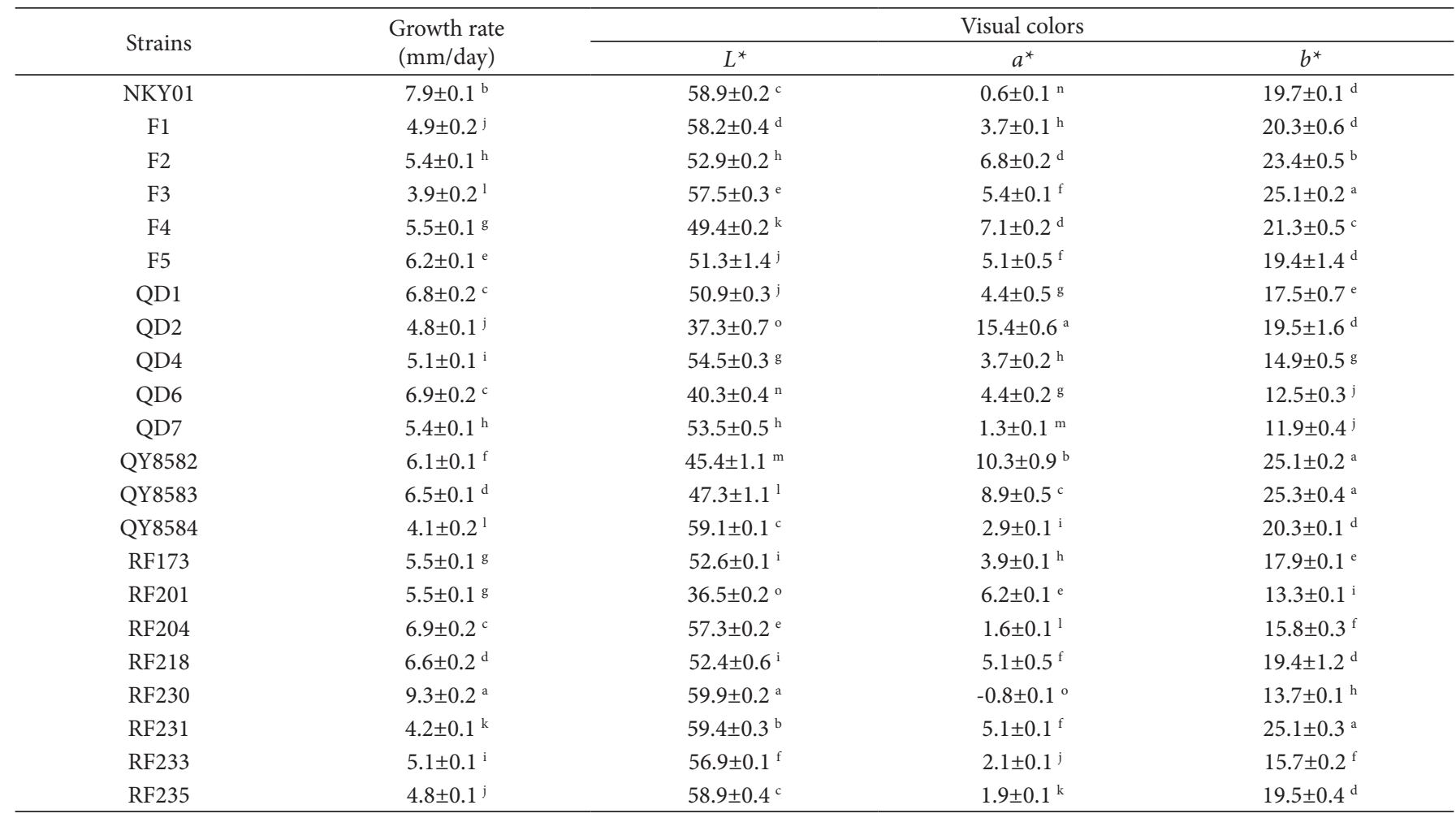

Data in same column with different superscript letter are significantly different at $p<0.05$.

among different strains. A. auricula RF201 and QD6 exhibited lower $L^{*}$ values (36.5 and 40.3), $a^{*}$ values (6.2 and 4.4) and $b^{*}$ values (13.3 and 12.5), indicating that their colony centers were dark with a little red and yellow colored. A. auricula QD2 possessed lower $L^{*}$ value (37.3) and the largest $a^{*}$ value (15.4), which implied that its colony center was dark red. A. auricula F3, QY8582, QY8583 and RF231 showed larger $b^{*}$ values (25.1, 25.1, 25.3 and 25.1), suggesting that their colony centers were yellow.

All A. auricula mycelia in this experiment secreted pigment during growth, whereas content of melanin pigments in PDA plate was positive correlated with $L^{\star}$ value in Hunter Lab color system (Fan et al., 2008). Therefore, according to $L^{\star}$ value in colony center, three A. auricula strains (RF201, QD2 and QD6) with higher pigment production capacity were selected for further study through submerged culture supplementing $1 \mathrm{~g} / \mathrm{L}$ l-tyrosine.

\subsection{Pigment production and mycelium biomass of A. auricula in fermentation broth}

The pigment production kinetic curves of $A$. auricula strains RF201, QD2 and QD6 in fermentation broth supplementing $1 \mathrm{~g} / \mathrm{L}$ 1-tyrosine are shown in Figure 2. As expected, the pigment yield quickly enhanced when incubation time was extended but it slowly reduced later. The maximal pigment yields were obtained on 6 or 7 days of incubation. Lagunas-Muñoz et al. (2006) reported that recombinant Escherichia coli W3110 could produce $6 \mathrm{~g} / \mathrm{L}$ melanin pigment on 3 days of incubation. However, E. coli was one of the major foodborne pathogens which could

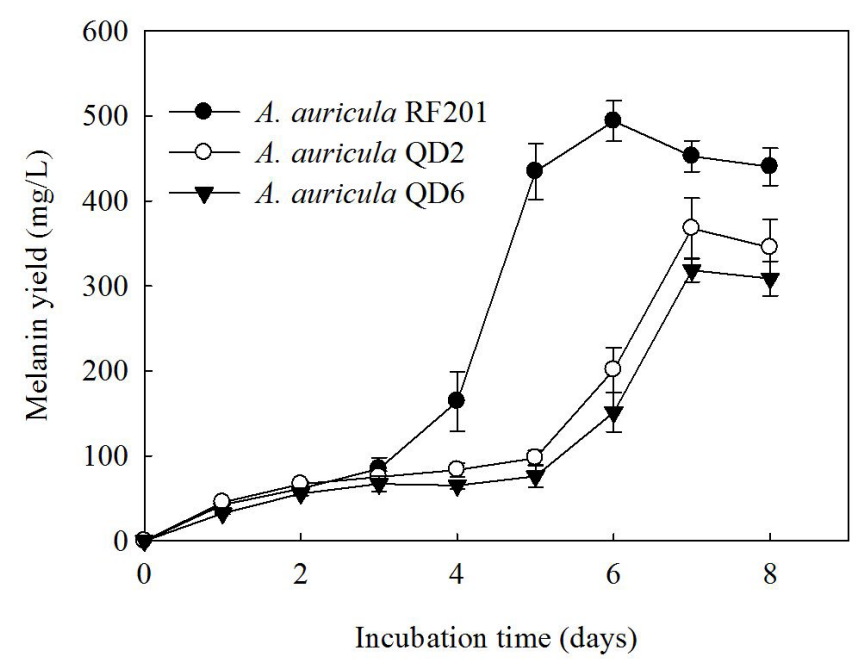

Figure 2. Pigment production kinetic curve of A. auricula strains in fermentation broth.

produce shiga toxin and its propagation might result in serious human diseases (Santos \& Stephanopoulos, 2008). Moreover, other hidden trouble and potential fatal danger might exist in metabolites of E. coli (Vu-Khac \& Cornick, 2008). A. auricula was a nontoxic macro-fungus and had been used as an edible mushroom for centuries in China. Melanin pigments produced by $A$. auricula were safe food colorants and could be applied to food processing. 
Table 2. Pigment yield and dry mycelium weight of $A$. auricula strains.

\begin{tabular}{cccc}
\hline Strains & Incubation time (days) & Pigment yield $(\mathrm{mg} / \mathrm{L})$ & Dry weight of mycelium $(\mathrm{g} / \mathrm{L})$ \\
\hline RF201 & 6 & $493.9 \pm 23.7^{\mathrm{a}}$ & $8.1 \pm 0.4^{\mathrm{a}}$ \\
QD2 & 7 & $367.6 \pm 35.8^{\mathrm{b}}$ & $6.3 \pm 0.4^{\mathrm{b}}$ \\
QD6 & 7 & $318.5 \pm 13.9^{\mathrm{c}}$ & $6.8 \pm 0.8^{\mathrm{b}}$ \\
\hline
\end{tabular}

Data in same column with different superscript letter are significantly different at $p<0.05$.

As shown in Table 2, the maximal pigment yield of $A$. auricula RF201, QD2 and QD6 were 493.9, 367.6 and $318.5 \mathrm{mg} / \mathrm{L}$ on 6,7 and 7 days of incubation, respectively. Among three strains, A. auricula RF201 possessed the strongest $(p<0.05)$ production ability of melanin pigments in the shortest incubation time. Meanwhile, dry weight of mycelium $(8.1 \mathrm{~g} / \mathrm{L})$ of A. auricula RF201 was the highest $(p<0.05)$ than those of other two $A$. auricula strains, which indicated that $A$. auricula RF201 possessed stronger growth and metabolism ability. The present study suggested that A. auricula RF201 could be used as potential excellent producer of melanin pigments.

\section{Conclusions}

Twenty-two $A$. auricula strains were screened for the strongest production ability of melanin pigments by solid culture and submerged culture. Comparing visual colors of colony center and growth rate of mycelium, A. auricula strains RF201, QD2 and QD6 were selected for further study. In fermentation broths, the maximal pigment yields of $A$. auricula RF201, QD2 and QD6 were 493.9, 367.6 and $318.5 \mathrm{mg} / \mathrm{L}$, respectively. The present study suggested that $A$. auricula RF201 was the strongest melanin-producing fungal strain for the potential use as an effective producer of natural melanin pigments.

\section{Acknowledgements}

This work was supported by Program for Liaoning Excellent Talents in University (No. LJQ2015031).

\section{References}

Chen, S. R., Jiang, B., Zheng, J. X., Xu, G. Y., Li, J. Y., \& Yang, N. (2008). Isolation and characterization of natural melanin derived from silky fowl (Gallus gallus domesticus Brisson). Food Chemistry, 111(3), 745-749. http://dx.doi.org/10.1016/j.foodchem.2008. 04.053.

Dalfard, A. B., Khajeh, K., Soudi, M. R., Naderi-Manesh, H., Ranjbar, B., \& Sajedi, R. H. (2006). Isolation and biochemical characterization of laccase and tyrosinase activities in a novel melanogenic soil bacterium. Enzyme and Microbial Technology, 39(7), 1409-1416. http://dx.doi.org/10.1016/j.enzmictec.2006.03.029.

Dong, C., \& Yao, Y. (2012). Isolation, characterization of melanin derived from Ophiocordyceps sinensis, an entomogenous fungus endemic to the Tibetan Plateau. Journal of Bioscience and Bioengineering, 113(4), 474-479. http://dx.doi.org//10.1016/j.jbiosc.2011. 12.001. PMid:22261188.

Fan, G., Han, Y., Gu, Z., \& Gu, F. (2008). Composition and colour stability of anthocyanins extracted from fermented purple sweet potato culture. Lebensmittel-Wissenschaft + Technologie, 41(8), 1412-1416. http://dx.doi.org/10.1016/j.lwt.2007.09.003.

Lagunas-Muñoz, V. H., Cabrera-Valladares, N., Bolívar, F., Gosset, G., \& Martínez, A. (2006). Optimum melanin production using recombinant Escherichia coli. Journal of Applied Microbiology, 101(5), 1002-1008. http://dx.doi.org/10.1111/j.1365-2672.2006.03013.x.

Liu, J. H., Tian, Y. G., Wang, Y., Nie, S. P., Xie, M. Y., Zhu, S., Wang, C. Y., \& Zhang, P. (2011). Characterization and in vitro antioxidation of papain hydrolysate from black-bone silky fowl (Gallus gallus domesticus Brisson) muscle and its fractions. Food Research International, 44(1), 133-138. http://dx.doi.org/10.1016/j.foodres.2010.10.050.

Santos, C. N. S., \& Stephanopoulos, G. (2008). Melanin-based highthroughput screen for l-tyrosine production in Escherichia coli. Applied and Environmental Microbiology, 74(4), 1190-1197. http:// dx.doi.org/10.1128/AEM.02448-07.

Sun, S., Zhang, X., Chen, W., Zhang, L., \& Zhu, H. (2016). Production of natural edible melanin by Auricularia auricula and its physicochemical properties. Food Chemistry, 196, 486-492. http://dx.doi.org/10.1016/j. foodchem.2015.09.069.

Tu, Y., Sun, Y., Tian, Y., Xie, M., \& Chen, J. (2009). Physicochemical characterisation and antioxidant activity of melanin from the muscles of Taihe Black-bone silky fowl (Gallus gallus domesticus Brisson). Food Chemistry, 114(4), 1345-1350. https://doi.org/10.1016/j. foodchem.2008.11.015.

Vu-Khac, H., \& Cornick, N. A. (2008). Prevalence and genetic profiles of Shiga toxin-producing Escherichia coli strains isolated from buffaloes, cattle, and goats in central Vietnam. Veterinary Microbiology, 126(4), 356-363. http://dx.doi.org/ 10.1016/j.vetmic.2007.07.023. PMid:17716835

Wang, H., Pan, Y., Tang, X., \& Huang, Z. (2006). Isolation and characterization of melanin from Osmanthus fragrans' seeds. Lebensmittel-Wissenschaft + Technologie, 39(5), 496-502. http:// dx.doi.org/10.1016/j.lwt.2005.04.001.

Wu, J., Ding, Z. Y., \& Zhang, K. C. (2006). Improvement of exopolysaccharide production by macro-fungus Auricularia auricula in submerged culture. Enzyme and Microbial Technology, 39(4), 743-749. http:// dx.doi.org/10.1016/j.enzmictec.2005.12.012.

Wu, Y., Shan, L., Yang, S., \& Ma, A. (2008). Identification and antioxidant activity of melanin isolated from Hypoxylon archeri, a companion fungus of Tremella fuciformis. Journal of Basic Microbiology, 48(3), 217-221. http://dx.doi.org/10.1002/jobm.200700366.

Xie, C., Gu, Z., You, X., Liu, G., Tan, Y., \& Zhang, H. (2010). Screening of edible mushrooms for release of ferulic acid from wheat bran by fermentation. Enzyme and Microbial Technology, 46(2), 125-128. http://dx.doi.org/10.1016/j.enzmictec.2009.10.005.

Zou, Y., \& Hou, X. (2017). Optimization of culture medium for production of melanin by Auricularia auricula. Food Science and Technology, 37(1), 153-157. http://dx.doi.org/10.1590/1678-457x.18016.

Zou, Y., Xie, C., Fang, G., Gu, Z., \& Han, Y. (2010). Optimization of ultrasound-assisted extraction of melanin from Auricularia auricula fruit bodies. Innovative Food Science \& Emerging Technologies, 11(4), 611-615. http://dx.doi.org/10.1016/j.ifset.2010.07.002.

Zou, Y., Zhao, Y., \& Hu, W. (2015). Chemical composition and radical scavenging activity of melanin from Auricularia auricula fruiting bodies. Food Science and Technology, 35(2), 253-258. http://dx.doi. org/10.1590/1678-457X.6482. 